\title{
Anidulafungin in children: Experience in a tertiary care children's hospital in Argentina
}

\author{
María T. Rosanova, M.D. ${ }^{a}$, Claudia Sarkis, M.D. ${ }^{a}$, Florencia Escarra, M.D. ${ }^{a}$, Carolina Epelbaum, M.D. ${ }^{a}$, \\ Norma Sberna, Pharmacists ${ }^{b}$, Susana Carnovale, M.D. ${ }^{c}$, Carlos Figueroa, M.D. ${ }^{d}$, Rosa Bologna, M.D. ${ }^{a}$ and \\ Roberto Lede, M.D. ${ }^{e}$
}

\begin{abstract}
Theexperience using anidulafungin for the treatment of invasive fungal infections in pediatrics is limited.

In this article, we describe our experience in 55 children. Anidulafungin was administered intravenously at a loading dose of $3 \mathrm{mg} / \mathrm{kg}$ once daily, followed by $1.5 \mathrm{mg} / \mathrm{kg}$ every 24 hours over a mean period of 14 days (range: 7-22 days). Patients' median age was 114 months old (interquartile range: 32-168 months old). All patients had underlying diseases. Among patients with bone marrow transplant, the difference in white blood cell count, transaminase levels, and renal function at baseline and at the end of anidulafungin administration was not significant. No adverse events were reported and no patient died from an anidulafungin-related cause. Anidulafungin may be considered an alternative for the prophylaxis or treatment of invasive fungal infections in pediatrics but methodologically robust studies are needed to confirm this.

Key words: anidulafungin, children, invasive fungal infections.

http:/ / dx.doi.org/10.5546/aap.2017.eng.374
\end{abstract}

To cite: Rosanova MT, Sarkis C, Escarra F, et al. Anidulafungin in children: Experience in a tertiary care children's hospital in Argentina Arch Argent Pediatr 2017;115(4):374-376.

\section{INTRODUCTION}

Invasive fungal infections (IFIs) are an increasingly more relevant health problem associated with a high rate of morbidity and mortality. ${ }^{1}$

Echinocandins, the latest class of antifungals introduced in the market, have a distinct mechanism of action: they act by inhibiting 1,3 beta-D glucan synthesis and damaging cell

a. Department of Infectious Diseases, Hospital de Pediatría "Prof. Dr. Juan P. Garrahan", Autonomous City of Buenos Aires, Argentina.

b. Pharmacy Department, Hospital dePediatría "Prof. Dr. Juan P. Garrahan", Autonomous City of Buenos Aires, Argentina.

c. Department of Microbiology, Hospital de Pediatría "Prof. Dr. Juan P. Garrahan", Autonomous City of Buenos Aires, Argentina.

d. Department of Bone Marrow Transplant, Hospital de Pediatría "Prof. Dr. Juan P. Garrahan", Autonomous City of Buenos Aires, Argentina.

e. Universidad Abierta Interamericana (UAI), Buenos Aires, Argentina.

E-mail address:

María T. Rosanova, M.D.: margris2@yahoo.com.ar

Funding: None.

Conflict of interest: None.

Received: 1-5-2017

Accepted: 2-21-2017 walls without affecting human cells because these lack 1,3 beta-D-glucan. ${ }^{2-5}$ These novel therapeutic options have been used in the adult population but experience in the pediatric setting, especially with anidulafungin, is limited.

Anidulafungin has been the latest echinocandin introduced in the market; its pharmacokinetic profile is different from that of the other echinocandins. It is not metabolized by the liver but undergoes more than $90 \%$ of slow degradation in plasma by nonspecific peptidases that open the molecular ring and form a substrate that is subjected to tertiary degradation by plasma proteases. ${ }^{5}$ Anidulafungin does not rely on renal excretion and does not interact with immunosuppressive agents. ${ }^{5-8}$

It shows an excellent in vitro activity against Candida spp. and Aspergillus spp., including fluconazole- and amphotericin B-resistant strains. ${ }^{1,6}$

Given anidulafungin's metabolism and excretion, no dose adjustment is required for patients with mild, moderate or severe liver or renal failure, turning it into an appealing drug for the prophylaxis and/or treatment of IFIs in transplant patients.

Mild or moderate infusion-related adverse effects were reported in children, including discomfort, facial erythema and flushing, fever, and low blood pressure. ${ }^{2}$

In spite of anidulafungin's favorable features, the experience in pediatrics is limited.

\section{OBJECTIVE}

The objective of this descriptive-prospective study was to describe our experience using anidulafungin in a pediatric population.

\section{MATERIAL AND METHODS}

In the setting of a public, tertiary care children's hospital, and given the temporary lack of lipid formulations of amphotericin, anidulafungin was indicated as prophylaxis or treatment between January and June of 2016 to 55 patients with proven, probable, or possible IFI, together with a follow-up protocol. Following an adequate bibliographic review, and after having assessed the lack of interactions and the scarce reports of 
adverse effects, the hospital's Drug Committee approved anidulafungin for its off-label use.

Lab tests: The monitoring of biochemical parameters included, at the beginning and end of treatment, white blood cell count (cells $/ \mathrm{mm}^{3}$ ), transaminase levels $(\mathrm{U} / \mathrm{L})$, bilirubin $(\mathrm{mg} / \mathrm{dL})$, and creatinine $(\mathrm{mg} / \mathrm{dL})$ in all patients.

Definitions: IFIs were defined according to the criteria established by the European Organization for Research and Treatment of Cancer/Invasive Fungal Infections Cooperative Group and the National Institute of Allergy and Infectious Diseases Mycoses Study Group (EORTC/MSG). ${ }^{9}$

Proven IFI: Positive histopathology based on a biopsy with associated tissue lesion or positive microbiological culture obtained from a sterile area with clinical or radiological features compatible with infection.

Probable IFI: Presence of a host factor plus a mycological criterion plus a clinical feature.

Possible IFI: Presence of host factors and clinical evidence of IFI, but absence of positive mycological criteria.

Dosage: Anidulafungin was administered intravenously at the recommended dose (loading dose of $3 \mathrm{mg} / \mathrm{kg}$ once daily, followed by $1.5 \mathrm{mg} /$ kg every 24 hours, both as prophylaxis and treatment). All possible or probable drug-related adverse events were recorded.

Data were processed using the Epi-Info 6.0 software. Continuous outcome measures were expressed as median and interquartile range (IQR) whereas categorical outcome measures, as absolute quantity and percent relative frequency.

\section{RESULTS}

All patients receiving anidulafungin were included (n: 55). Their median age was 114 months old (IQR: 32-168 months old). All patients had underlying diseases; bone marrow transplant (29 patients, 53\%), liver transplant (9 patients, 16\%), and other hematooncological diseases (7 patients, $13 \%$ ) were the most common ones (see Table 1).

Treatment was administered over a mean period of 14 days (IQR: 7-22 days) and indicated as treatment for 27 patients $(49 \%)$ and as prophylaxis for 28 patients $(51 \%)$. IFIs were confirmed in 10 patients, and Candida albicans was the most commonly isolated fungus (Table 1).

Bone marrow transplant recipients accounted for the largest number of patients; mean values of biochemical parameters in this group were analyzed at the beginning and end of treatment. These included transaminase levels: $29.5 \mathrm{U} / \mathrm{L}$ and $32 \mathrm{U} / \mathrm{L}$ ( $p: 0.44$ ); bilirubin: 0.35 and $0.30 \mathrm{mg} / \mathrm{dL}$ ( $p$ : $0.20)$; and creatinine: 0.52 and $0.60 \mathrm{mg} / \mathrm{dL}(p: 0.67)$. A wide variability was observed in white blood cell count on account of underlying diseases; however, the difference between the baseline value and that at the end of drug administration was not significant: median of 2810 cells $/ \mathrm{mm}^{3}$ and 5160 cells $/ \mathrm{mm}^{3}$, respectively ( $p: 0.07$ ) (see Table 2).

In terms of effectiveness, it was observed that IFIs resolved in $100 \%$ of patients treated with anidulafungin, and that $100 \%$ of patients receiving the drug as prophylaxis did not develop an IFI in the 30-day follow-up.

No mild, moderate or severe adverse events were reported and no patient died from an

TABLE 1. Characteristics of patients ( $n: 55)$

\begin{tabular}{ll}
\hline Outcome measure & \\
\hline Age in months, median (IQR) & $114(32-168)$ \\
Underlying disease, $\mathrm{n}(\%)$ & 55 patients $(100 \%)$ \\
$\quad$ Bone marrow transplant & 29 \\
$\quad$ Hematooncological disease & 7 \\
$\quad$ Liver transplant & 9 \\
$\quad$ Other & 10 \\
Immunosuppresive therapy, $\mathrm{n}(\%)$ & 50 patients $(91 \%)$ \\
Anidulafungin indication & \\
$\quad$ Prophylaxis, $\mathrm{n}(\%)$ & $29(53 \%)$ \\
$\quad$ Treatment, $\mathrm{n}(\%)$ & $26(47 \%)$ \\
Documented fungus & 10 patients $(18 \%)$ \\
$\quad$ C. albicans (blood culture) & 5 patients \\
$\quad$ C. parapsilosis (blood culture) & 1 paciente \\
$\quad$ Aspergillus flavus (bronchoalveolar lavage) & 2 patients \\
Trichosporon asahii (urine culture) & 2 patients \\
Treatment duration & \\
$\quad$ Median (IQR) & 14 días $(7-22)$ \\
\hline
\end{tabular}

IQR: interquartile range. 
anidulafungin-related cause. Follow-up at 30 days was complete, and no relapse or mortality associated with anidulafungin use was observed.

\section{DISCUSSION}

This study started due to the temporary lack of lipid formulations of amphotericin in our hospital. Anidulafungin use was based on the hypothesis that it may be better tolerated and that its effectiveness may not be inferior to that of other prophylaxis or treatment options. Its indication also considered that, unlike other antifungal agents, there were no known interactions between anidulafungin and immunosupressors, thus favoring its use in transplant patients, and that infusion-related adverse effects were less common and posed a lower cost compared to lipid formulations of amphotericin. ${ }^{1,5,10-12}$

In a phase II study, anidulafungin was administered to 24 children between 2 and 17 years old at a dose of 0.75 or $1.5 \mathrm{mg} / \mathrm{kg}$ of body weight per day; mild or moderate adverse effects were reported but only four cases were classified as possibly or probably drug-related. ${ }^{2}$ These included discomfort, facial erythema and flushing, increased urea, fever, and low blood pressure, which were not observed in this series. These results demonstrated that bone marrow transplant recipients did not require discontinuing anidulafungin use due to adverse effects. Particularly, no cases of severe nephrotoxicity or hepatotoxicity directly attributed to drug use were reported. Transaminase levels remained stable or reduced during treatment.

White blood cell count varied in the studied population on account of patients' underlying disease; no significant alterations similar to those reported in the literature were confirmed. ${ }^{2}$

Either when indicated as prophylaxis or treatment, the success rates observed with anidulafungin were indicative of sufficient effectiveness, as reported in other patient series, especially adult patients. ${ }^{1,11,13-15}$

The limited literature regarding the pediatric population supports our findings on the safety and effectiveness of anidulafungin, and highlights the need for further studies to strengthen these statements. ${ }^{1-3}$

\section{CONCLUSIONS}

These findings warrant the need to plan methodologically adequate studies to prove the hypothesis that anidulafungin may be a valid alternative for the prophylaxis or treatment of IFIs in children.

\section{REFERENCES}

1. Wilke MW. Invasive Fungal Infections in Infants- Focus on Anidulafungin. Clin Med Insights Pediatr 2013;7:7-11.

2. GobernadoM, Cantón E. Anidulafungina.Rev Esp Quimioter 2008;21(2):99-114.

3. Benjamin DK Jr, Driscoll T, Seibel NL, et al. Safety and pharmacokinetics of intravenous anidulafungin in children with neutropenia at high risk for invasive fungal infections. Antimicrob Agents Chemother 2006;50(2):632-8.

4. Dowell JA, Stogniew M, Krause D, et al. Anidulafungin does not require dosage adjustment in subjects with varying degrees of hepatic or renal impairment. J Clin Pharmacol 2007;47(4):461-70.

5. Reboli AC, Rotstein C, Pappas PG, et al. Anidulafungin versus fluconazole for invasive candidiasis. $N$ Engl J Med 2007;356(24):2472-82.

6. Acuña C. Anidulafungina. Actualización microbiológica y clínica. Drugs of Today 2008;44(Suppl 2):1-25.

7. Catalán González M, Montejo González JC. Nueva aportación en terapia antifúngica: Anidulafungina. Aspectos farmacológicos de la anidulafungina. Rev Iberoam Micol 2008;25:92-100.

8. Dowell JA, Stogniew M, Krause D, et al. Lack of pharmacokinetic interaction between anidulafungin and tacrolimus. J Clin Pharmacol 2007;47(3):305-14.

9. De Pauw B, Walsh TJ, Donnelly JP, et al. Revised definitions of invasive fungal disease from the European Organization for Research and Treatment of Cancer/Invasive Fungal Infections Cooperative Group and the National Institute of Allergy and Infectious Diseases Mycoses Study Group (EORTC/MSG) Consensus Group. Clin Infect Dis 2008;46(12):1813-21.

10. Denning DW. Echinocandin antifungal drugs. Lancet 2003;362(9390):1142-51.

11. Tapısiz A. Anidulafungin: is it a promising option in the treatment of pediatric invasive fungal infections? Expert Rev Anti Infect Ther 2011;9(3):339-46.

12. Pappas PG, Kauffman CA, Andes DR, et al. Clinical Practice Guideline for the Management of Candidiasis: 2016Update by the Infectious Diseases Society of America. Clin Infect Dis 2016;62(4):e1-50.

13. Liu $P$, Ruhnke $M$, Meersseman $W$, et al. Pharmacokinetics of anidulafungin in critically ill patients with candidemia/ invasive candidiasis. Antimicrob Agents Chemother 2013;57(4):1672-6.

14. Ghannoum M, D'Angelo M. Anidulafungin: A potent antifungal that targets Candida and Aspergillus. Infect Dis Clin Pract 2005;13(4):165-78.

15. Azanza Perea JR. Equinocandinas: aspectos aplicados de la farmacología. Rev Iberoam Micol 2016;33(3):140-4.

TABLE 2. Course of biochemical parameters among patients with bone marrow transplant treated with anidulafungin (n: 29 patients)

\begin{tabular}{lccc}
\hline Biochemical parameters & Treatment initiation (median) & End of treatment (median) & $\boldsymbol{P}$ \\
\hline Transaminase levels (IU / L) & 29.5 & 32 & 0.44 \\
Bilirubin $(\mathrm{mg} / \mathrm{dL})$ & 0.35 & 0.30 & 0.20 \\
Creatinine $(\mathrm{mg} / \mathrm{dL})$ & 0.52 & 0.60 & 0.67 \\
White blood cell count (cells $/ \mathrm{mm}^{3}$ ) & 2810 & 5160 & 0.07 \\
\hline
\end{tabular}

